Explicit/Implicit, Conservative Domain

Decomposition Procedures for

Parabolic Problems Based on

Block-centered Finite Differences

Clint N. Dawson

and

Todd F. Dupont

November, 1991

TR91-37 



\title{
EXPLICIT/IMPLICIT, CONSERVATIVE DOMAIN \\ DECOMPOSITION PROCEDURES FOR PARABOLIC PROBLEMS \\ BASED ON BLOCK-CENTERED FINITE DIFFERENCES
}

\author{
CLINT N. DAWSON* ${ }^{*}$ AND TODD F. DUPONT ${ }^{\dagger}$
}

\begin{abstract}
Domain decomposition procedures for solving parabolic equations are considered. The underlying discretization is block-centered finite differences. In these procedures, fluxes at subdomain interfaces are calculated from the solution at the previous time level. These fluxes serve as Neumann boundary data for implicit, block-centered discretizations in the subdomains. A priori error estimates are derived, and numerical results examining the stability, accuracy, and parallelism of the scheme are presented.
\end{abstract}

Keywords: Domain decomposition, parabolic equations, block-centered finite differences, mixed finite elements, parallel computing.

AMS(MOS) Subject Classification: 65M60, 65P05

1. Introduction. In this paper, we present a domain decomposition procedure for parabolic partial differential equations, where the underlying discretization is block-centered finite differences. Block-centered finite differences are widely used in applications, and are especially useful for problems in rectangular geometries where approximations to both solution values and fluxes are desired. Unlike point-centered finite differences, the block-centered method gives solution values and fluxes to the same order of accuracy.

* Department of Mathematical Sciences, Rice University, Houston, TX
$f_{\text {Department of Computer Science, University of Chicago, Chicago, IL }}$ 
For parabolic equations, it is often useful to use implicit time-stepping, due to the severe time step constraint which arises from explicit time-stepping. Thus, a global system of equations must be solved at each time step. Domain decomposition can be used to divide the global problem into smaller subdomain problems, which can be solved in parallel. The difficulty lies in piecing the subdomain solutions together into a reasonable approximation to the true solution. In the block-centered finite difference approach, solution values are approximated by constants at grid block centers, and fluxes are approximated on the block edges. In the domain decomposition procedure presented here, the interfaces between subdomains coincide with certain grid block edges. At the beginning of each time step, interface fluxes are calculated using the solution from the previous time step. These fluxes then serve as Neumann boundary data for implicit subdomain problems. We examine ways of calculating the interface fluxes so that no iterations between edges and subdomains are necessary. Hence, this domain decomposition approach should be nearly optimal, in the sense that the speed-up obtained is roughly equal to the number of subdomains. Furthermore, the method is easy to incorporate into existing implicit codes. As we will see, the explicit nature of the flux calculation gives rise to a constraint involving the time step and an interface discretization parameter; however, this constraint is much less severe than the constraint needed for a fully explicit scheme.

It has been shown in $[6,7]$ that block-centered finite differences can be derived from the mixed finite element method, using the lowest-order Raviart-Thomas [5] approximating spaces, and special quadrature rules. The procedures defined here can 
be extended to higher-order Raviart-Thomas spaces on rectangles; however, we will deal only with the lowest order case here.

Galerkin domain decomposition techniques were developed in $[1,2,3]$. In $[1,2]$, finite element and point-centered finite difference domain decomposition procedures were analyzed; these methods used Dirichlet boundary data on subdomain interfaces. These approaches are of optimal order in error but have the disadvantage that they are not globally conservative. In [3], we defined conservative procedures based on Galerkin finite elements which use Neumann boundary data at interfaces. Here we use a related approach to treat block-centered finite differences. Although the approach is similar the details are quite different.

In the next section, we describe the domain decomposition approach in a onedimensional setting, and derive an error estimate. In Section 3, we extend the procedures to the case of two subdomains in two space dimensions. In Section 4, we examine numerically the stability, accuracy, and parallelism of the scheme on certain test problems. The numerical rates of convergence for the two-dimensional test problems are slightly better than what we have been able to prove in general. However, they agree with the one-dimensional results derived in Section 2.

2. One Space Dimension. We begin with a one-dimensional example. Let $u(x, t)$ satisfy the heat equation:

$$
\begin{aligned}
u_{t}-u_{x x}=0, \quad 0<x<1, \quad 0<t \leq T, \\
u_{x}(0, t)=u_{x}(1, t)=0, \quad 0<t \leq T,
\end{aligned}
$$




$$
u(x, 0)=u^{0}(x), \quad 0<x<1 .
$$

Define

$$
q(x, t)=-u_{x}(x, t) .
$$

Then

$$
q(0, t)=q(1, t)=0, \quad 0<t \leq T,
$$

and

$$
u_{t}+q_{x}=0
$$

Let $(\cdot, \cdot)$ and $\|\cdot\|$ represent the $L^{2}(0,1)$ inner product and norm, respectively. Let $H_{0}^{1}(0,1)$ denote the intersection of the Sobolev space $H^{1}(0,1)$ with the functions which vanish at zero and one. Multiplying (2.4) by a function $v \in H_{0}^{1}(0,1)$ and integrating by parts we obtain

$$
(q(\cdot, t), v)-\left(u(\cdot, t), v_{x}\right)=0 .
$$

Multiplying (2.6) by a function $w \in L^{2}(0,1)$ and integrating, we find

$$
\left(u_{t}(\cdot, t), w\right)+\left(q_{x}(\cdot, t), w\right)=0 .
$$

Let $\delta_{x}$ be a partition of $(0,1)$ :

$$
\delta_{x}: 0=x_{1 / 2}<x_{3 / 2}<\ldots<x_{I+1 / 2}=1 \text {, }
$$


and set

$$
\begin{gathered}
x_{i}=\frac{x_{i-1 / 2}+x_{i+1 / 2}}{2}, \\
h_{i}^{x}=x_{i+1 / 2}-x_{i-1 / 2}, \\
h_{i+1 / 2}^{x}=x_{i+1}-x_{i}, \\
\Omega_{i}^{x}=\left[x_{i-1 / 2}, x_{i+1 / 2}\right] .
\end{gathered}
$$

Let $h=\max _{i} h_{i}^{x}$. For functions $f(x), g(x)$, let $f_{k}=f\left(x_{k}\right), f_{k+1 / 2}=f\left(x_{k+1 / 2}\right)$, and define the discrete inner product

$$
\langle f, g\rangle=\sum_{i=0}^{I} f_{i+1 / 2} g_{i+1 / 2} h_{i+1 / 2}^{x},
$$

and the corresponding semi-norm

$$
\|f f\|^{2}=\langle f, f\rangle .
$$

As mentioned in the introduction, block-centered finite differences can be derived from the mixed finite element method using the lowest-order Raviart-Thomas approximating spaces and special quadrature rules. Denote by $\mathcal{M}_{-1}\left(d ; h^{x}\right)$ the finite dimensional subspace of $L^{2}(0,1)$ consisting of all functions which are polynomials of degree at most $d$ on each interval $\Omega_{i}^{x}$. For $r \geq 0$, let $\mathcal{M}_{r}\left(d ; h^{x}\right)=\mathcal{M}_{-1}\left(d ; h^{x}\right) \cap \mathcal{C}^{r}(0,1)$, and

$$
\mathcal{M}_{r}^{0}\left(d ; h^{x}\right)=\mathcal{M}_{r}\left(d ; h^{x}\right) \cap\{v(x) \mid v(0)=v(1)=0\} .
$$

Let $\mathcal{Q}, \mathcal{U}$ be the lowest order Raviart-Thomas approximating spaces; i.e., $\mathcal{Q}=$ $\mathcal{M}_{1}^{0}\left(1 ; h^{x}\right)$ and $\mathcal{U}=\mathcal{M}_{-1}\left(0 ; h^{x}\right)$. The dimensions of $\mathcal{Q}$ and $\mathcal{U}$ are $I-1$ and $I$, respectively. By using a standard nodal basis, namely, the linear "chapeau" functions in $x$, 
a function $v \in \mathcal{Q}$ is determined by its values at the points $x_{i+1 / 2}, i=1, \ldots, I-1$. A function $w \in \mathcal{U}$ is piecewise constant on $\Omega_{i}^{x}, i=1, \ldots, I$. Denote this constant by $w_{i}$.

Assume a decomposition of $(0,1)$ into two intervals, $\Omega_{1}=(0, \bar{x})$ and $\Omega_{2}=(\bar{x}, 1)$, where $\bar{x}=x_{\vec{k}+1 / 2} \in \delta_{x}$ for some integer $\bar{k}, 0<\bar{k}<I$. Let $0<H \leq \min (\bar{x}, 1-\bar{x})$, and assume $\bar{x}-H, \bar{x}+H$ are also in $\delta_{x}$.

For a given smooth function $\psi(x)$, let $B(\psi)$ denote an approximation to $-\psi_{x}(\bar{x})$, determined by

$$
B(\psi)=\frac{1}{H} \int_{0}^{1} \phi^{\prime}(x) \psi(x) d x
$$

where $\phi \in \mathcal{Q}$,

$$
\phi(x)= \begin{cases}\frac{x-\bar{x}+H}{H}, & \bar{x}-H \leq x \leq \bar{x}, \\ \frac{\bar{x}+H-x}{\bar{H}}, & \bar{x} \leq x \leq \bar{x}+H \\ 0, & \text { otherwise. }\end{cases}
$$

It is easily seen that, for $\psi$ thrice differentiable in $x$,

$$
\left|\psi_{x}(\bar{x})+B(\psi)\right| \leq C H^{2}
$$

A block centered finite difference-domain decomposition procedure can be defined as follows. Let $0=t^{0}<t^{1}<\ldots<t^{M}=T$ be a given sequence, $\Delta t^{n}=t^{n}-t^{n-1}$, and for $f=f(t)$, let $f^{n}=f\left(t^{n}\right)$, and

$$
\partial_{t} f^{n}=\frac{f^{n}-f^{n-1}}{\Delta t^{n}}
$$

Assume $U^{n-1} \in \mathcal{U}, U^{n-1} \approx u^{n-1}$, is given. Let $q^{n} \approx Q^{n} \in \mathcal{Q}$. First, approximate $q^{n}(\bar{x})$ by $Q^{n}(\bar{x})$, where

$$
Q^{n}(\bar{x}) \equiv Q_{\bar{k}+1 / 2}^{n}=B\left(U^{n-1}\right)
$$


Enforce the boundary condition $(2.2)$ by

$$
Q_{1 / 2}^{n}=Q_{I+1 / 2}^{n}=0
$$

For $1 \leq i \leq I-1, i \neq \bar{k}$, approximate $q_{i+1 / 2}^{n}$ by

$$
Q_{i+1 / 2}^{n}=\frac{U_{i+1}^{n}-U_{i}^{n}}{h_{i+1 / 2}^{x}}
$$

and approximate $u_{i}^{n}$ by $U_{i}^{n}$, where

$$
\partial_{t} U_{i}^{n}+\frac{Q_{i+1 / 2}^{n}-Q_{i-1 / 2}^{n}}{h_{i}^{x}}=0, \quad i=1, \ldots, I .
$$

Substituting (2.12), (2.13), and (2.14) into (2.15) where appropriate, we obtain a positive definite, symmetric system of equations for determining $U^{n}$. Since the flux $Q_{\bar{k}+1 / 2}^{n}$ is determined independently of $U^{n}$, the resulting system of equations decouples into two disjoint sets of equations, corresponding to the subdomains $\Omega_{1}$ and $\Omega_{2}$. These systems can be solved in parallel.

This procedure is conservative in the following sense. Multiplying (2.15) by $h_{i}^{x}$, summing on $i$, and applying (2.13), we find that $\left(U^{n}, 1\right)=\left(U^{n-1}, 1\right)$, which the true solution also satisfies.

The initial condition (2.3) is enforced by setting

$$
U^{0}=\tilde{u}^{0}
$$

where $\tilde{u}^{0}$ is the $L^{2}$-projection of $u^{0}$ into $\mathcal{U}$; that is,

$$
\left(u^{0}-\tilde{u}^{0}, w\right)=0, \quad w \in \mathcal{U}
$$


Equations (2.14)-(2.15) are equivalent to the following system of equations:

$$
\begin{gathered}
\left\langle Q^{n}, v\right\rangle-\left(U^{n}, v_{x}\right)=0, \quad v \in \overline{\mathcal{Q}}, \\
\left(\partial_{t} U^{n}, w\right)+\left(Q_{x}^{n}, w\right)=0, \quad w \in \mathcal{U},
\end{gathered}
$$

where $\overline{\mathcal{Q}}=\mathcal{Q} \cap\{v \mid v(\bar{x})=0\}$.

We now state an error estimate for the method given by (2.12)-(2.16).

Theorem 2.1. Let $\tilde{u}(\cdot, t)$ denote the $L^{2}$ projection of $u(\cdot, t)$ into $\mathcal{U}$. Assume $u$ is sufficiently smooth, and

$$
\frac{\Delta t}{H^{2}} \leq \frac{1}{4},
$$

where $\Delta t=\max _{n} \Delta t^{n}$. Then, there exists a constant $C$, independent of $h, \Delta t$, and $H$, such that,

$$
\left(\sum_{n=1}^{M}\|\| q^{n}-Q^{n} \|\left.\right|^{2} \Delta t^{n}\right)^{1 / 2}+\max _{n}\left\|\tilde{u}^{n}-U^{n}\right\| \leq C\left(\Delta t+h^{2}+H^{3}\right)
$$

Proof. Let $\bar{Q}(\cdot, t) \in \mathcal{Q}$ denote the interpolant of $q(\cdot, t)$; that is,

$$
\bar{Q}\left(x_{i+1 / 2}, t\right)=q\left(x_{i+1 / 2}, t\right), \quad i=0, \ldots, I .
$$

Note that

$$
\left(\bar{Q}_{x}(\cdot, t), w\right)=\left(q_{x}(\cdot, t), w\right)=-\left(u_{t}(\cdot, t), w\right), \quad w \in \mathcal{U} .
$$

Define $\bar{U}(\cdot, t) \in \mathcal{U}$ for each $t \in[0, T]$ by

$$
\bar{U}\left(x_{1}, t\right)=u(0, t),
$$




$$
\begin{gathered}
\bar{Q}(\bar{x}, t)=B(\bar{U}(\cdot, t)), \\
\langle\bar{Q}(\cdot, t), v\rangle-\left(\bar{U}(\cdot, t), v_{x}\right)=0, \quad v \in \overline{\mathcal{Q}} .
\end{gathered}
$$

The system (2.25), (2.26) represents $I-1$ equations in $I-1$ unknowns, and it is easily shown that $\bar{U}(\cdot, t)$ is unique.

Set $\mu=Q-\bar{Q}, \hat{\mu}(x)=\mu(\bar{x}) \phi(x)$, and $\xi=U-\tilde{U}$. Since $\phi \in \mathcal{M}_{0}^{0}\left(1 ; h^{x}\right), \hat{\mu}$ is in this space as well. Substract (2.26) from (2.18), and (2.23) from (2.19) to obtain

$$
\begin{array}{r}
\left\langle\mu^{n}, v\right\rangle-\left(\xi^{n}, v_{x}\right)=0, \quad v \in \overline{\mathcal{Q}}, \\
\left(\partial_{t} \xi^{n}, w\right)+\left(\mu_{x}^{n}, w\right)=\left(\partial_{t}\left(\tilde{u}^{n}-\bar{U}^{n}\right), w\right)+\left(u_{t}^{n}-\partial_{t} u^{n}, w\right), \quad w \in \mathcal{U} .
\end{array}
$$

By (2.12), (2.25), (2.9), and (2.22),

$$
\begin{aligned}
\mu^{n}(\bar{x}) & =Q^{n}(\bar{x})-\bar{Q}^{n}(\bar{x}) \\
& =B\left(\xi^{n-1}\right)-\left(\bar{Q}^{n}(\bar{x})-\bar{Q}^{n-1}(\bar{x})\right) \\
& =\frac{1}{H}\left(\xi^{n-1}, \phi_{x}\right)-\left(q^{n}(\bar{x})-q^{n-1}(\bar{x})\right) .
\end{aligned}
$$

Set $v=\mu^{n}-\hat{\mu}^{n}$ in (2.27), $w=\xi^{n}$ in (2.28), multiply (2.29) by $H \mu^{n}(\bar{x})$, and add the resulting equations to obtain

$$
\begin{aligned}
\left(\partial_{t} \xi^{n}, \xi^{n}\right) & +\left.\left\|\mu^{n}\right\|\right|^{2}+H\left|\mu^{n}(\bar{x})\right|^{2} \\
= & \left\langle\mu^{n}, \hat{\mu}^{n}\right\rangle+\left(\partial_{t}\left(\tilde{u}^{n}-\bar{U}^{n}\right), \xi^{n}\right)+\left(u_{t}^{n}-\partial_{t} u^{n}, \xi^{n}\right) \\
& +\left(\xi^{n-1}-\xi^{n}, \hat{\mu}_{x}^{n}\right)-H\left(q^{n}(\bar{x})-q^{n-1}(\bar{x})\right) \hat{\mu}^{n}(\bar{x}) . \\
\leq & \frac{3}{4}\left|\left\|\mu^{n}\left|\left\|^{2}+\frac{1}{3}\right\|\left\|\hat{\mu}^{n}\right\|\right|^{2}+C\right\| \partial_{t}\left(\tilde{u}^{n}-\bar{U}^{n}\right)\left\|^{2}+C\right\| \xi^{n} \|^{2}\right. \\
& +C\left\|u_{t}^{n}-\partial_{t} u^{n}\right\|^{2}+\frac{1}{2 \Delta t^{n}}\left\|\xi^{n-1}-\xi^{n}\right\|^{2}+\frac{\Delta t^{n}}{2}\left\|\hat{\mu}_{x}^{n}\right\|^{2} \\
& +C H\left|q^{n}(\bar{x})-q^{n-1}(\bar{x})\right|^{2}+\epsilon H\left|\hat{\mu}^{n}(\bar{x})\right|^{2}, \\
9 &
\end{aligned}
$$


where $\epsilon$ is a small positive constant. In (2.30) we have applied the well-known inequality,

$$
a b \leq \frac{\epsilon^{\prime}}{2} a^{2}+\frac{1}{2 \epsilon^{\prime}} b^{2}, \quad a, b, \epsilon^{\prime} \in \mathrm{R}, \quad \epsilon^{\prime}>0,
$$

for different choices of $\epsilon^{\prime}$.

Note that

$$
||\left|\hat{\mu}^{n}\right|||^{2} \leq 2 H\left|\mu^{n}(\bar{x})\right|^{2}
$$

and

$$
\left\|\hat{\mu}_{x}^{n}\right\|^{2}=\frac{2}{H^{2}}\left|\mu^{n}(\bar{x})\right|^{2}
$$

Thus, choosing $\epsilon=\frac{1}{12}$, and enforcing the inequality (2.20) we find

$$
\begin{aligned}
\left.\frac{1}{3}\left|\left\|\left.\hat{\mu}^{n}||\right|^{2}+\frac{\Delta t^{n}}{2}\right\| \hat{\mu}_{x}^{n} \|^{2}+\epsilon H\right| \hat{\mu}^{n}(\bar{x})\right|^{2} & \leq\left(\frac{2}{3}+\frac{\Delta t^{n}}{H^{2}}+\epsilon\right) H\left|\mu^{n}(\bar{x})\right|^{2} \\
& \leq H\left|\mu^{n}(\bar{x})\right|^{2} .
\end{aligned}
$$

For $q$ and $u$ sufficiently smooth,

$$
H\left|q^{n}(\bar{x})-q^{n-1}(\bar{x})\right|^{2}=\mathcal{O}\left(H \Delta t^{2}\right),
$$

and

$$
\left\|u_{t}^{n}-\partial_{t} u^{n}\right\|^{2}=\mathcal{O}\left(\Delta t^{2}\right)
$$

Furthermore,

$$
\left(\partial_{t} \xi^{n}, \xi^{n}\right)=\frac{1}{2 \Delta t^{n}}\left[\left\|\xi^{n}\right\|^{2}-\left\|\xi^{n-1}\right\|^{2}+\left\|\xi^{n}-\xi^{n-1}\right\|^{2}\right]
$$


Combine (2.30), (2.31)-(2.34) to find

$$
\frac{1}{2 \Delta t^{n}}\left[\left\|\xi^{n}\right\|^{2}-\left\|\xi^{n-1}\right\|^{2}\right]+\frac{1}{4}\left\|\mu^{n}\right\|\left\|^{2} \leq C \Delta t^{2}+C\right\| \partial_{t}\left(\tilde{u}^{n}-\bar{U}^{n}\right) \|^{2}
$$

Next, we estimate $\partial_{t}\left(\tilde{u}^{n}-\bar{U}^{n}\right)$. For $0 \leq t \leq T$, let $U^{e}(\cdot, t) \in \mathcal{U}$ be defined by

$$
\begin{gathered}
U^{e}\left(x_{1}, t\right)=u(0, t), \\
\langle\bar{Q}(\cdot, t), v\rangle-\left(U^{e}(\cdot, t), v_{x}\right)=0, \quad v \in \mathcal{Q} .
\end{gathered}
$$

Drop $t$ dependence momentarily. Let $\nu=\bar{U}-U^{e}$, and subtract (2.37) from (2.26) to obtain

$$
\left(\nu, v_{x}\right)=0, \quad v \in \overline{\mathcal{Q}}
$$

Since $\bar{U}\left(x_{1}\right)=U^{e}\left(x_{1}\right), \nu\left(x_{1}\right)=0$. By $(2.38)$,

$$
\nu_{i}=0, \quad i \leq \bar{k}
$$

and

$$
\nu_{i}=\nu_{\bar{k}+1}, \quad i \geq \bar{k}+1
$$

Since $\bar{Q}(\bar{x})=q(\bar{x})$, by $(2.22)$,

$$
\begin{aligned}
0 & =B(\bar{U})-\bar{Q}(\bar{x}) \\
& =B(\nu)+B\left(U^{e}\right)-q(\bar{x})
\end{aligned}
$$

Thus,

$$
\begin{aligned}
B(\nu) & =-\left(B\left(U^{e}\right)-q(\bar{x})\right) \\
& =-\left[B\left(U^{e}-\tilde{u}\right)+B(\tilde{u})-q(\bar{x})\right] \\
& \equiv \rho .
\end{aligned}
$$


By (2.39) and (2.40),

$$
B(\nu)=-\frac{1}{H} \nu_{\vec{k}+1},
$$

Thus,

$$
\left|\nu_{\bar{k}+1}\right|=H|\rho| .
$$

In [7], it is shown that $\left|U^{e}\left(x_{i}\right)-\tilde{u}\left(x_{i}\right)\right|=\mathcal{O}\left(h^{2}\right)$. Thus,

$$
B\left(U^{e}-\tilde{u}\right)=\mathcal{O}\left(\frac{h^{2}}{H}\right) .
$$

Since $\phi_{x} \in \mathcal{U}, B(u)=B(\tilde{u})$, and by $(2.11),|B(\tilde{u})-q(\bar{x})|=\mathcal{O}\left(H^{2}\right)$. Combine these results to find

$$
|\rho|=\mathcal{O}\left(\frac{h^{2}}{H}+H^{2}\right)
$$

Thus

$$
\left|\nu_{\bar{k}+1}\right|=\mathcal{O}\left(h^{2}+H^{3}\right)
$$

and, by $(2.39),(2.40)$,

$$
\left|\nu\left(x_{i}\right)\right|=\mathcal{O}\left(h^{2}+H^{3}\right), \quad i=1, \ldots, I .
$$

By the triangle inequality,

$$
\left|\tilde{u}\left(x_{i}\right)-\bar{U}\left(x_{i}\right)\right| \leq\left|\tilde{u}\left(x_{i}\right)-U^{e}\left(x_{i}\right)\right|+\left|\nu\left(x_{i}\right)\right|=\mathcal{O}\left(h^{2}+H^{3}\right) .
$$

Differencing (2.22), (2.36) and (2.37) in time, the arguments in [7] can be used to show

$$
\left|\partial_{t}\left(\tilde{u}^{n}\left(x_{i}\right)-U^{e, n}\left(x_{i}\right)\right)\right|=\mathcal{O}\left(h^{2}\right), \quad i=1, \ldots, I .
$$


Thus, differencing (2.24)-(2.26) in time, and following the same arguments as given above, the estimate $(2.46)$ holds with $\nu$ replaced by $\partial_{t} \nu^{n}$. Hence,

$$
\left|\partial_{t}\left(\tilde{u}^{n}\left(x_{i}\right)-\bar{U}^{n}\left(x_{i}\right)\right)\right|=\mathcal{O}\left(h^{2}+H^{3}\right)
$$

Substitute (2.49) into (2.35), multiply by $2 \Delta t^{n}$, sum on $n$, and apply Gronwall's Lemma, to obtain

$$
\left(\sum_{n=1}^{M}\|\| \mu^{n}\|\|^{2} \Delta t^{n}\right)^{1 / 2}+\max _{n}\left\|\xi^{n}\right\| \leq C\left(\Delta t+h^{2}+H^{3}\right)
$$

Apply this result together with the triangle inequality and (2.22) to complete the proof of the Theorem.

3. Extension to two dimensions. In this section, we extend the scheme presented above to a more general equation in two space dimensions. The extension to three space dimensions and higher is straightforward.

Let $\Omega=(0,1) \times(0,1)$. Assume that $u^{0}, a$, and $b$ are smooth, real-valued functions on $\bar{\Omega}$, with $a=\operatorname{diag}\left(a^{x}, a^{y}\right)$. Assume $b$ is nonnegative, and positive constants $a_{0}^{x}, a_{1}^{x}$, $a_{0}^{y}$, and $a_{1}^{y}$ exist such that

$$
a_{0}^{x} \leq a^{x}(x, y) \leq a_{1}^{x}
$$

and

$$
a_{0}^{y} \leq a^{y}(x, y) \leq a_{1}^{y}
$$

For some $T>0$, let $u(x, y, t)$ satisfy

$$
\frac{\partial u}{\partial t}-\nabla \cdot(a \nabla u)+b u=0, \quad \text { on } \Omega \times(0, T] \text {, }
$$




$$
\begin{array}{r}
\frac{\partial u}{\partial n_{\Omega}}=0, \quad \text { on } \partial \Omega \times(0, T], \\
u(x, 0)=u^{0}(x), \quad \text { on } \Omega,
\end{array}
$$

where $n_{\Omega}$ is the outward normal to $\partial \Omega$. Let $q$ denote the vector

$$
q=\left(q^{x}, q^{y}\right)=-\left(a^{x} u_{x}, a^{y} u_{y}\right) .
$$

Then, by (3.1),

$$
\frac{\partial u}{\partial t}+\nabla \cdot q+b u=0, \quad \text { on } \Omega \times(0, T]
$$

and, by $(3.2)$,

$$
q \cdot n_{\Omega}=0, \quad \text { on } \partial \Omega \times(0, T] .
$$

Let $\delta_{x}$ and $\delta_{y}$ be partitions of $(0,1)$ :

$$
\begin{aligned}
& \delta_{x}: 0=x_{1 / 2}<x_{3 / 2}<\ldots<x_{I+1 / 2}=1, \\
& \delta_{y}: 0=y_{1 / 2}<y_{3 / 2}<\ldots<y_{J+1 / 2}=1,
\end{aligned}
$$

and define $x_{i}, h_{i}^{x}, h_{i+1 / 2}^{x}$, and $\Omega_{i}^{x}$ as in the last section, with similar definitions for $y_{j}$, $h_{j}^{y}, h_{j+1 / 2}^{y}$, and $\Omega_{j}^{y}, j=1, \ldots, J$. Let $h^{x}=\max _{i} h_{i}^{x}, h^{y}=\max _{j} h_{j}^{y}, h=\max \left(h^{x}, h^{y}\right)$, and $\Omega_{i, j}=\Omega_{i}^{x} \times \Omega_{j}^{y}$.

For $f(x, y)$ and $g(x, y)$, let $f_{i, j}=f\left(x_{i}, y_{j}\right), f_{i+1 / 2, j}=f\left(x_{i+1 / 2}, y_{j}\right)$ and $f_{i, j+1 / 2}=$ $f\left(x_{i}, y_{j+1 / 2}\right)$. Define the discrete inner products:

$$
\begin{aligned}
&\langle f, g\rangle_{x, a^{x}}=\sum_{j=1}^{J} \sum_{i=0}^{I} \frac{1}{a_{i+1 / 2, j}^{x}} f_{i+1 / 2, j} g_{i+1 / 2, j} h_{i+1 / 2}^{x} h_{j}^{y}, \\
&\langle f, g\rangle_{y, a^{y}}=\sum_{j=0}^{J} \sum_{i=1}^{I} \frac{1}{a_{i, j+1 / 2}^{y}} f_{i, j+1 / 2} g_{i, j+1 / 2} h_{i}^{x} h_{j+1 / 2}^{y} \\
& 14
\end{aligned}
$$


and the corresponding seminorms

$$
\begin{aligned}
& \|f \mid\|_{x, a^{x}}^{2}=\langle f, f\rangle_{x, a^{x}} \\
& \|f \mid\|_{y, a^{y}}^{2}=\langle f, f\rangle_{y, a^{y}} .
\end{aligned}
$$

Let $\langle f, g\rangle_{x},\left\||| f|\||_{x}\right.$ denote $\langle f, g\rangle_{x, a^{x}}$ and $\left|\left\|f|\||_{x, a^{x}}\right.\right.$ with $a^{x} \equiv 1$, similarly for $\langle f, g\rangle_{y}$ and ||$|f| \|_{y}$. Note that \|\|$f \mid \|_{x}\left(\|\| f|| \|_{y}\right)$ and $\||| f \mid\|_{x, a^{x}}\left(\left|\left\|f|\||_{y, a^{y}}\right)\right.\right.$ are equivalent.

Let $\mathcal{Q}, \mathcal{U}$ be the lowest order Raviart-Thomas approximating spaces defined on the tensor-product mesh $\delta_{x} \otimes \delta_{y}$; that is, $\mathcal{Q}=\mathcal{Q}^{x} \times \mathcal{Q}^{y}$, where

$$
\begin{aligned}
& \mathcal{Q}^{x}=\mathcal{M}_{0}^{0}\left(1 ; h^{x}\right) \otimes \mathcal{M}_{-1}\left(0 ; h^{y}\right), \\
& \mathcal{Q}^{y}=\mathcal{M}_{-1}\left(0 ; h^{x}\right) \otimes \mathcal{M}_{0}^{0}\left(1 ; h^{y}\right),
\end{aligned}
$$

and

$$
\mathcal{U}=\mathcal{M}_{-1}\left(0 ; h^{x}\right) \otimes \mathcal{M}_{-1}\left(0 ; h^{y}\right)
$$

The dimensions of $\mathcal{Q}^{x}, \mathcal{Q}^{y}$, and $\mathcal{U}$ are $(I-1) \cdot J, I \cdot(J-1)$, and $I \cdot J$, respectively. In this case, a function $v^{x} \in \mathcal{Q}^{x}$ is determined by its values at the points $\left(x_{i+1 / 2}, y_{j}\right)$, $i=1, \ldots, I-1, j=1, \ldots, J$. Similarly, a function $v^{y} \in \mathcal{Q}^{y}$ is determined by its values at $\left(x_{i}, y_{j+1 / 2}\right), i=1, \ldots, I, j=1, \ldots, J-1$. A function $w \in \mathcal{U}$ is piecewise constant on $\Omega_{i, j}, i=1, \ldots, I, j=1, \ldots, J$. Denote this constant by $w_{i, j}$.

Assume a decomposition of $\Omega$ into two strips, $\Omega_{1}=(0, \bar{x}) \times(0,1)$ and $\Omega_{2}=$ $(\bar{x}, 1) \times(0,1)$, where $\bar{x}=x_{\bar{k}+1 / 2} \in \delta_{x}$ for some integer $\bar{k}, 0<\bar{k}<I$. Let $0<H \leq$ $\min (\bar{x}, 1-\bar{x})$, and assume $\bar{x}-H, \bar{x}+H$ are also in $\delta_{x}$. 
Similar to one space dimension, define

$$
B(\psi(\cdot, y))=\frac{1}{H} \int_{0}^{1} \phi^{\prime}(x) \psi(x, y) d x \approx-\psi_{x}(\bar{x}, y)
$$

where $\phi$ is given by $(2.10)$.

A domain decomposition procedure can be defined as follows. Assume $U^{n-1} \in \mathcal{U}$ is given. Let $Q^{n}=\left(Q^{x, n}, Q^{y, n}\right) \in \mathcal{Q}$. First, approximate $q^{x, n}\left(\ddot{x}, y_{j}\right)$ by $Q^{x, n}\left(\bar{x}, y_{j}\right)$ :

$$
Q^{x, n}\left(\bar{x}, y_{j}\right) \equiv Q_{\bar{k}+1 / 2, j}^{x, n}=-a^{x}\left(\bar{x}, y_{j}\right) B\left(U^{n-1}\left(\cdot, y_{j}\right)\right), \quad j=1, \ldots, J
$$

Enforce the boundary condition (3.6) by setting

$$
Q_{1 / 2, j}^{x, n}=Q_{I+1 / 2, j}^{x, n}=0, \quad j=1, \ldots, J
$$

and

$$
Q_{i, 1 / 2}^{y, n}=Q_{i, J+1 / 2}^{y, n}=0, \quad i=1, \ldots, I .
$$

For $j=1, \ldots, J, 1 \leq i \leq I-1, i \neq \bar{k}$, approximate $q_{i+1 / 2, j}^{x, n}$ by

$$
Q_{i+1 / 2, j}^{x, n}=-a_{i+1 / 2, j}^{x} \frac{U_{i+1, j}^{n}-U_{i, j}^{n}}{h_{i+1 / 2}^{x}}
$$

For $i=1, \ldots, I, j=1, \ldots, J-1$, approximate $q_{i, j+1 / 2}^{y, n}$ by

$$
Q_{i, j+1 / 2}^{y, n}=-a_{i, j+1 / 2}^{y} \frac{U_{i, j+1}^{n}-U_{i, j}^{n}}{h_{j+1 / 2}^{y}}
$$

and approximate $u_{i, j}^{n}$ by $U_{i, j}^{n}$, where

$$
\partial_{t} U_{i, j}^{n}+\frac{Q_{i+1 / 2, j}^{x, n}-Q_{i-1 / 2, j}^{x, n}}{h_{i}^{x}}+\frac{Q_{i, j+1 / 2}^{y, n}-Q_{i, j-1 / 2}^{y, n}}{h_{j}^{y}}+b_{i, j} U_{i, j}^{n}=0
$$


Substituting (3.8), (3.9), (3.10), (3.11), and (3.12) into (3.13), we obtain a positive definite, symmetric system of equations for determining $U^{n}$. Since the flux $Q_{\bar{k}+1 / 2, j}^{x, n}$ is determined independently of $U^{n}$, the resulting system of equations decouples into two disjoint sets of equations, corresponding to the subdomains $\Omega_{1}$ and $\Omega_{2}$. These systems can be solved in parallel.

The initial condition is enforced by setting

$$
U^{0}=\tilde{u}^{0}
$$

where $\tilde{u}^{0}$ is the $L^{2}$-projection of $u^{0}$ into $\mathcal{U}$.

Equations (3.11)-(3.13) are equivalent to the following system of equations:

$$
\begin{array}{r}
\left\langle Q^{x, n}, v^{x}\right\rangle_{x, a^{x}}+\left\langle Q^{y, n}, v^{y}\right\rangle_{y, a^{y}}-\left(U^{n}, \nabla \cdot v\right)=0, \quad v \in \overline{\mathcal{Q}} \\
\left(\partial_{t} U^{n}, w\right)+\left(\nabla \cdot Q^{n}, w\right)+\left(U^{n}, w\right)=0, \quad w \in \mathcal{U},
\end{array}
$$

where

$$
\begin{aligned}
\overline{\mathcal{Q}} & =\overline{\mathcal{Q}}^{x} \times \mathcal{Q}^{y} \\
\overline{\mathcal{Q}}^{x} & =\mathcal{Q}^{x} \cap\left\{v^{x} \mid v^{x}(\bar{x}, y)=0\right\} .
\end{aligned}
$$

The extension of the one-dimensional arguments given in the last section to two space dimensions requires an assumption on the truncation error term $\rho$ which is difficult to verify. Based on experimental results, the rate of convergence given in Theorem 1 may hold in two space dimensions as well. We have not been able to prove this in general, however. An estimate which is slightly less sharp is stated below. 
Define $\bar{Q} \in \mathcal{Q}, \bar{U} \in \mathcal{U}$ to be the elliptic block-centered finite difference approximations to $q$ and $u[7] ;$ that is, for each $t \in[0, T]$,

$$
\begin{array}{r}
\left\langle\bar{Q}^{x}(\cdot, t), v^{x}\right\rangle_{x, a^{x}}+\left\langle\bar{Q}^{y}(\cdot, t), v^{y}\right\rangle_{y, a^{y}}-(\bar{U}(\cdot, t), \nabla \cdot v)=0, \quad v \in \mathcal{Q}, \\
(\nabla \cdot \bar{Q}(\cdot, t), w)+(b \bar{U}(\cdot, t), w)=-\left(u_{t}(\cdot, t), w\right), \quad w \in \mathcal{U}
\end{array}
$$

Then, by extensions of Theorems 4.1,4.2, and (5.27) in [7] to the case $b>0$,

$$
\left\|\left|\bar{Q}^{x}-q^{x}\right|\right\|_{x}+\left\|\left|\bar{Q}^{y}-q^{y}\right|\right\|_{y}+\|\bar{U}-\tilde{u}\|+\left\|\partial_{t}\left(\bar{U}^{n}-\tilde{u}^{n}\right)\right\| \leq C h^{2}
$$

where $\tilde{u}^{n}$ is the $L^{2}$-projection of $u^{n}$ into $\mathcal{U}$. Let $\kappa=q-\bar{Q}$. The following theorem is proven in [4].

Theorem 3.1. Assume $u, a^{x}, a^{y}, b$, and $u^{0}$ are suffciently smooth, and

$$
\frac{\Delta t}{H^{2}} \max _{j} a^{x}\left(\bar{x}, y_{j}\right) \leq \frac{1}{4}
$$

where $\Delta t=\max _{n} \Delta t^{n}$. Then, there exists a constant $C$, independent of $h, \Delta t$, and $H$, such that,

$$
\begin{aligned}
\left(\sum_{n=1}^{M}[\right. & {\left[\left\|q^{x, n}-Q^{x, n}\left|\left\|_{x}^{2}+\right\|\left\|q^{y, n}-Q^{y, n} \mid\right\|_{y}^{2}\right] \Delta t^{n}\right)^{1 / 2}+\max _{n}\left\|\tilde{u}^{n}-U^{n}\right\|\right.} \\
\leq & C\left(\Delta t H^{1 / 2}+H^{2.5}+H^{-1 / 2} h^{2}+H\left[\sum_{n=1}^{M} \sum_{j=1}^{J}\left|\kappa_{\bar{k}+1 / 2, j}^{x, n}\right|^{2} h_{j}^{y} \Delta t^{n}\right]^{1 / 2}\right) \\
& +C\left(h^{2}+\Delta t\right) .
\end{aligned}
$$

The fully implicit block-centered finite difference sheme, analyzed in [7], has a convergence rate of $\mathcal{O}\left(h^{2}+\Delta t\right)$. Compared to this estimate, (3.21) appears to be 
suboptimal by a half power of $H$. This result still represents a "superconvergent" estimate, however, since one would expect to do no better than $\mathcal{O}(h+\Delta t)$ when approximating by piecewise constants. In certain cases, the $H^{-1 / 2} h^{2}$ term can be improved to $H^{1 / 2} h^{2}$, depending on the mesh. In particular, this is the case if the mesh is symmetric about $\bar{x}$ in the interval $[\bar{x}-H, \bar{x}+H]$, or if it is "translation invariant in $H$ " in this interval. By "translation invariant in $H$ " we mean that the mesh in the interval $[\bar{x}, \bar{x}+H]$ can be obtained by adding $H$ to each mesh point in the interval $[\bar{x}-H, \bar{x}]$. The fourth term on the right side of (3.21) is at worst $\mathcal{O}\left(H h^{3 / 2}\right)$, by (3.19).

By using different integration kernels in (3.7), one can obtain approximations to the normal derivative on the interface which are higher order in $H$. For example, choose $H=\min (\bar{x} / 2,(1-\bar{x}) / 2)$, and assume $\bar{x}, \bar{x} \pm H$, and $\bar{x} \pm 2 H$ are in the partition $\delta_{x}$. Define $\phi=\phi_{4}((x-\bar{x}) / H)$, where

$$
\phi_{4}(x)= \begin{cases}(x-2) / 12, & 1 \leq x \leq 2, \\ -5 x / 4+7 / 6, & 0 \leq x \leq 1, \\ 5 x / 4+7 / 6, & -1 \leq x \leq 0, \\ -(x+2) / 12, & -2 \leq x \leq-1, \\ 0, & \text { otherwise. }\end{cases}
$$

This choice of $\phi$ in (3.7) gives a $\mathcal{O}\left(H^{4}\right)$ approximation to the derivative. An algorithm which is second order in time may also be derived, using a second order backward time difference. These and other extensions of the method are discussed in [4]. 


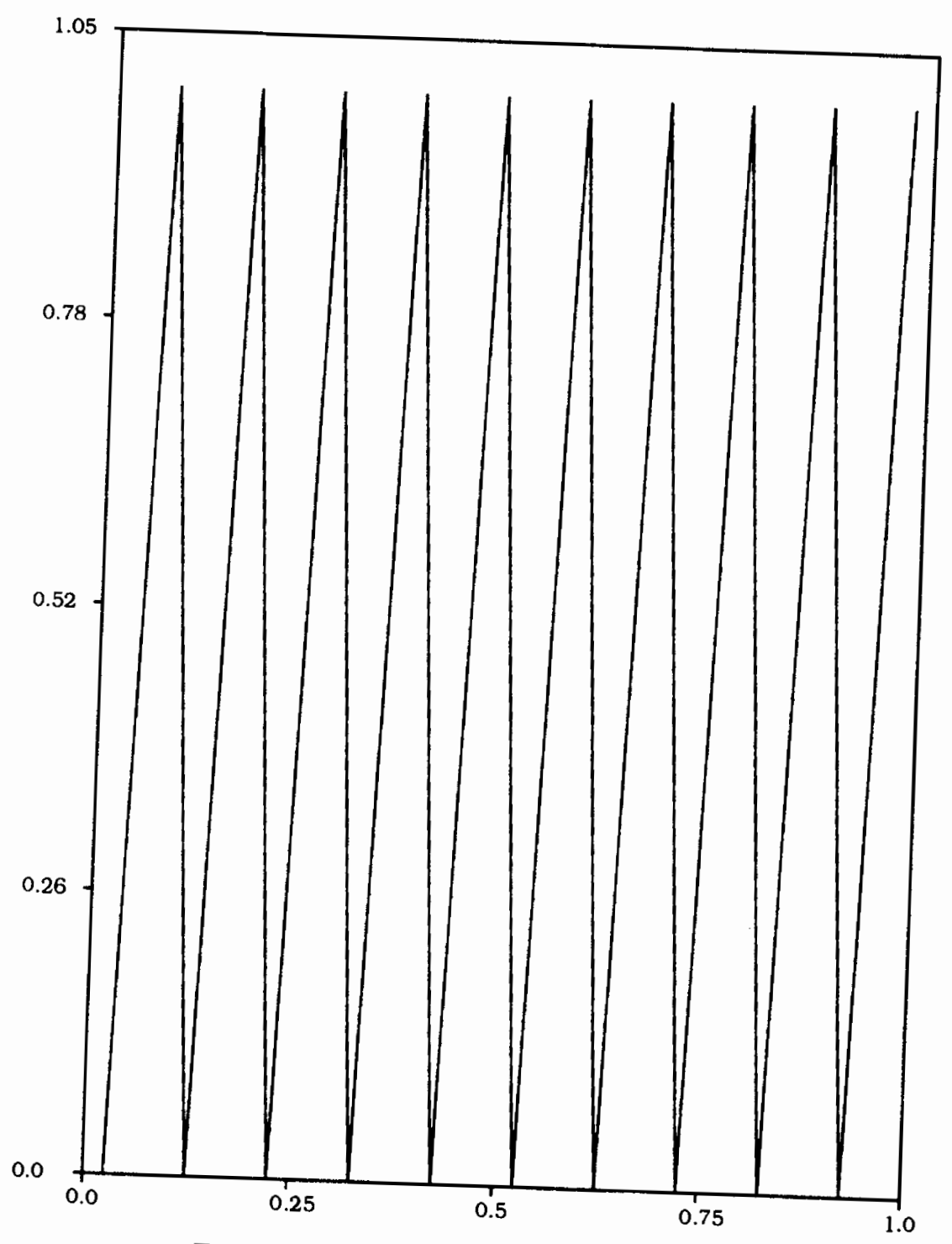

FIG. 1. Initial condition for stability test

4. Numerical results. In this section, we present numerical results examining the stability, accuracy, and parallelism of the scheme described above.

First, we examine the stability of the scheme as it relates to the time step constraint (3.20). We consider the algorithm (3.8)-(3.13), with $a=\operatorname{diag}(1,1), b=0$, and with initial data given in Figure 1. In Figure 2, we plot $\|U(\cdot, t)\|$ versus $t$ for different values of $\Delta t / H^{2}$. As can be seen in this figure, when the constraint is violated by as much as a factor of 3 , the $L^{2}$ norm of $U$ blows up with time. 


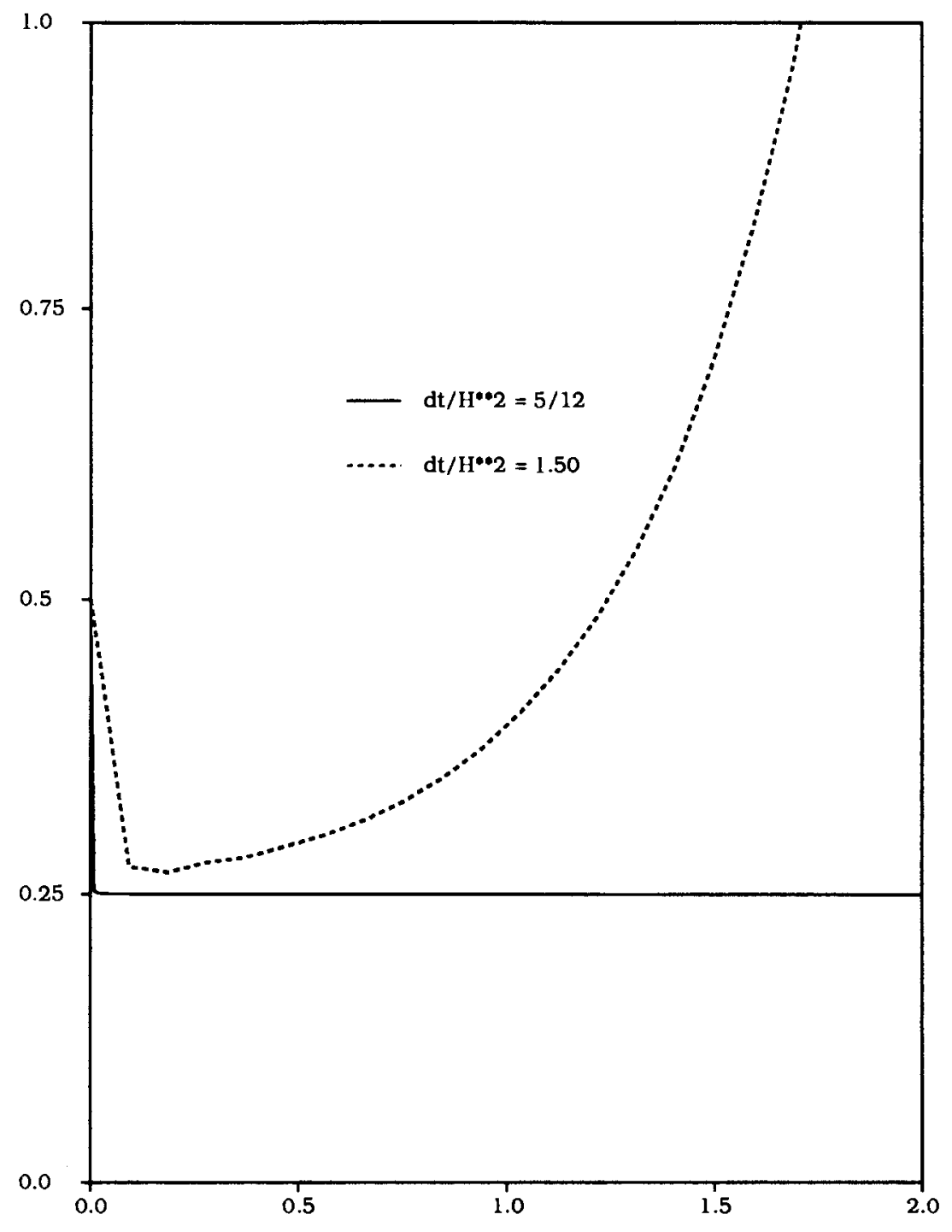

FIG. 2. Plot of $\|U\|$ versus $t$ 
Next, we examine the errors in the solution and the diffusive flux for two test problems. Let $\Omega=(0,1)^{2}$, and consider the problem

$$
\begin{array}{rc}
u_{t}-\Delta u=0, & (x, y) \in \Omega, t \in(0, T], \\
u^{0}(x, y)=\cos (2 \pi x) \cos (\pi y), & (x, y) \in \Omega, \\
\frac{\partial u}{\partial n_{\Omega}}=0, & t \in(0, T],
\end{array}
$$

which has true solution $u(x, y, t) \equiv u_{1}(x, y, t)=e^{-5 \pi^{2} t} \cos (2 \pi x) \cos (\pi y)$. We will look at three scenarios. Scenario 1 is fully implicit block-centered finite differences (no domain decomposition). Scenarios 2 and 3 involve domain decomposition with uniform and nonuniform mesh. We give the details of each case below.

Scenarios:

1. Fully implicit block-centered finite differences on uniform mesh $h ; \Delta t=4 h^{2}$.

2. Global uniform mesh; two subdomains $\Omega_{1}=(0, .5) \times(0,1), \Omega_{2}=(.5,1) \times(0,1)$, with $H=3 h, \Delta t=4 h^{2}$.

3. Two subdomains $\Omega_{1}=(0, .4) \times(0,1), \Omega_{2}=(.4,1) \times(0,1)$. The coarsest mesh on $\Omega_{1}$ consists of 5 blocks in the $x$-direction and 20 blocks in the $y$-direction. On $\Omega_{2}$, the coarsest mesh has 15 blocks in the $x$-direction and 20 in the $y$-direction. All subsequent mesh refinements are obtained by halving this mesh. Moreover, defining $h_{i}^{x}$ to be the mesh spacing in the $x$-direction for subdomain $i, i=1,2$, then $H=3 h_{1}^{x}$ and $\Delta t=4\left(h_{1}^{x}\right)^{2}$.

The errors in the solution for Scenarios 1-3 for this problem are compared in Table 1. Here $e_{h}=\|(U-\tilde{u})\|$ at $T=.1$. Three mesh refinements were used, and an 


\begin{tabular}{||r|c|c|c|c|c|c||}
\multicolumn{2}{c}{ Scenario 1 } & \multicolumn{3}{c}{ Scenario 2 } & \multicolumn{3}{c||}{ Scenario 3 } \\
\hline$h^{-1}$, & $e_{h} * 10^{4}$ & Rate & $e_{h} * 10^{4}$ & Rate & $e_{h} * 10^{4}$ & Rate \\
\hline 20 & 56.7 & - & 65.1 & - & 55.8 & - \\
\hline 40 & 12.0 & - & 11.97 & - & 11.78 & - \\
\hline 80 & 2.87 & 2.15 & 2.73 & 2.28 & 2.8 & 2.61 \\
\hline
\end{tabular}

\begin{tabular}{l}
\multicolumn{2}{c}{ Scenario 1 } \\
\begin{tabular}{||c|c|c|c|c|c|c||} 
Scenario 2 & \multicolumn{3}{c}{ Scenario 3 } \\
\hline$h^{-1}$, & $\gamma_{h} * 10^{3}$ & Rate & $\gamma_{h} * 10^{3}$ & Rate & $\gamma_{h} * 10^{3}$ & Rate \\
\hline 20 & 54.9 & - & 51.9 & - & 67.0 & - \\
\hline 40 & 15.0 & - & 14.2 & - & 17.5 & - \\
\hline 80 & 3.87 & 1.91 & 3.63 & 1.92 & 4.30 & 1.98 \\
\hline
\end{tabular} \\
Convergence of diffusive flux: $u(x, t)=u_{1}(x, y, t)$
\end{tabular}

experimental rate of convergence was calculated using a least squares fit of the data. As can be seen in this table, the errors for each scenario are roughly of the same order of magnitude, and the errors appear to be $\mathcal{O}\left(h^{2}\right)$ in each case. This can be predicted from the proof of Theorem 2 in Scenario 2, but is better than what is predicted for Scenario 3 , where nonuniform mesh is used.

The errors in the diffusive flux are given in Table 2. Here

$$
\gamma_{h}=\left(\sum_{n}\left[\|\| q^{x, n}-Q^{x, n}\left|\left\|_{x}^{2}+\right\|\left\|q^{y, n}-Q^{y, n}\right\|\right|_{y}^{2}\right] \Delta t\right)^{\frac{1}{2}} .
$$

We see virtually the same phenomena in Table 2 as in Table 1 . The errors are all of roughly the same magnitude, and converge like $h^{2}$. 
Next, consider

$$
\begin{aligned}
u_{t}-\Delta u & =f, & (x, y) \in \Omega, & t \in(0, T], \\
u^{0}(x, y) & =0, & & (x, y) \in \Omega, \\
\frac{\partial u}{\partial n_{\Omega}} & =0, & & t \in(0, T],
\end{aligned}
$$

with $f$ chosen so that $u(x, y, t)=u_{2}(x, y, t)=100 t x^{3}(1-x)^{2} \cos (2 \pi y)$. We consider the Scenarios 1-3 above, as well as a fourth scenario, which is simply the fully implicit scheme on the same grid used in Scenario 3. The errors in solution values and the diffusive flux are given in Tables 3 and 4, respectively. Here we see that on the coarser grids, the fully implicit solutions (Scenarios 1 and 4) have smaller error than the domain decomposition solutions (Scenarios 2 and 3). As the mesh is refined, the errors in the domain decomposition solutions drop dramatically, so that on the finest mesh, these errors are comparable in size to the fully implicit errors. This is not surprising; heuristically, one would expect the domain decomposition solution to approach the fully implicit solution as $h, H$, and $\Delta t$ approach zero. Because the errors decrease so rapidly in the domain decomposition cases, the experimental rates of convergence are substantially higher than predicted by Theorem 2 .

The method has been implemented for problems in two and three space dimensions on an Intel iPSC/860 Hypercube with 32 processors, located at Rice University. As an example of the performance consider the problem

$$
\begin{array}{r}
u_{t}-\nabla \cdot(a \nabla u)=f, \quad(x, y, z) \in(0,1)^{3}, \quad t \in(0, T], \\
u^{0}(x, y, z)=\cos (\pi x) \cos (\pi y) \cos (\pi z), \quad(x, y, z) \in(0,1)^{3},
\end{array}
$$


$\begin{array}{llll}\text { Scenario } 1 & \text { Scenario } 2 & \text { Scenario } 3 & \text { Scenario } 4\end{array}$

\begin{tabular}{||r|c|c|c|c|c|c|c|c||}
\hline$h^{-1}$, & $e_{h} * 10^{4}$ & Rate & $e_{h} * 10^{4}$ & Rate & $e_{h} * 10^{4}$ & Rate & $e_{h} * 10^{4}$ & Rate \\
\hline 20 & 11.8 & - & 24.2 & - & 65.8 & - & 10.6 & - \\
\hline 40 & 2.85 & - & 4.45 & - & 10.9 & - & 2.56 & - \\
\hline 80 & .738 & - & .891 & - & 1.73 & - & .661 & - \\
\hline 160 & .183 & 2.00 & .20 & 2.31 & .29 & 2.61 & .17 & 2.00 \\
\hline
\end{tabular}

TABLE 3

Convergence of solution: $u(x, t)=u_{2}(x, y, t)$

Scenario $1 \quad$ Scenario $2 \quad$ Scenario $3 \quad$ Scenario 4

\begin{tabular}{||r|c|c|c|c|c|c|c|c||}
\hline$h^{-1}$, & $\gamma_{h} * 10^{3}$ & Rate & $\gamma_{h} * 10^{3}$ & Rate & $\gamma_{h} * 10^{3}$ & Rate & $\gamma_{h} * 10^{3}$ & Rate \\
\hline 20 & .777 & - & 7.02 & - & 16.9 & - & .870 & - \\
\hline 40 & .171 & - & 1.03 & - & 2.76 & - & .194 & - \\
\hline 80 & .044 & - & .138 & - & .393 & - & .050 & - \\
\hline 160 & .011 & 2.04 & .019 & 2.84 & .053 & 2.78 & .012 & 2.04 \\
\hline
\end{tabular}

Convergence of diffusive flux: $u(x, t)=u_{2}(x, y, t)$ 


$$
\frac{\partial u}{\partial n_{\Omega}}=0, \quad t \in(0, T]
$$

with $a=.005\left(1+e^{2 x} e^{3 y} e^{z}\right)$, and $f$ chosen so that $u(x, y, t)=e^{-2 \pi^{2} t} u^{0}(x, y, z)$. In Table 5, we present timings and global $L^{2}$ errors for different domain decompositions applied to this problem. In these runs a uniform mesh was used with 20 grid blocks in each direction. The timestep $\Delta t=.005, T=.10$, and $H=.20$. A diagonally preconditioned conjugate gradient algorithm was used to solve the linear systems on each subdomain. In Table 5 , the notation $k \times m \times n$ refers to domain decomposition with $k$ domains in the $x$-direction, $m$ in the $y$-direction, and $n$ in the $z$-direction. When $k=m=n=1$, this represents the fully implicit solution. For these runs, $k * m * n$ processors were used.

The table shows that, for this test problem, the domain decomposition algorithm produced results which were slighly more accurate than the fully implicit scheme, and the amount of clock time needed to solve the problem decreased essentially linearly with the number of processors used. In fact, in some cases the speed-up is slightly better than linear, due to the fact that the iterative linear solver converged in fewer iterations. These results also indicate that the time needed to calculate the interface derivatives and pass this information to neighboring subdomains is small relative to the time needed to solve subdomain problems.

5. Conclusion. In conclusion, we have presented a domain decomposition approach for solving parabolic equations using an explicit/implicit, block-centered finite difference discretization. A priori error estimates have been derived for a one- 
dimensional version of the scheme, and error estimates have been stated for a twodimensional version of the scheme. The one dimensional results appear to be sharp, while the two-dimensional results are slightly suboptimal. Numerical experiments indicate that the scheme performs quite well and is highly parallelizable.

6. Acknowledgments. The authors would like to thank the National Science Foundation Center for Research on Parallel Computation for the use of their computational facilities.

\section{REFERENCES}

[1] Dawson, C. N. and Q. Du, A finite element domain decomposition method for parabolic equations, Rice Technical Report TR90-21, Dept. of Mathematical Sciences, Rice University.

[2] Dawson, C. N., Q. Du, and T. F. Dupont, A finite difference domain decomposition procedure for numerical solution of the heat equation, University of Chicago Dept. of Computer Science Tech. Report 89-09, to appear in Math. Comp.

[3] Dawson, C. N. and T. F. Dupont, Explicit/implicit, conservative domain decomposition procedures for parabolic problems in one space dimension, Rice Technical Report TR90-26, Dept. of Mathematical Sciences, Rice University, to appear in Math. Comp.

[4] Dawson, C. N. and T. F. Dupont, Analysis of explicit/implicit, block-centered finite difference domain decomposition procedures for parabolic problems in multidimensions, Rice Technical Report TR91-36, Dept. of Mathematical Sciences, Rice University.

[5] Raviart, P. A. and J. M. Thomas, A mixed finite element method for 2nd order elliptic problems, in Mathematical Aspects of the Finite Element Method, Rome, 1975, Lecture Notes in Mathematics, Springer-Verlag, Berlin, 1977.

[6] Russell, T. F. and M. F. Wheeler, Finite element and finite difference methods for continuous flow problems, in The Mathematics of Reservoir Simulation (R. E. Ewing, ed.), Frontiers 
in Science, Society for Industrial and Applied Mathematics, Philadelphia, 1983.

[7] Weiser, A. and M. F. Wheeler, On convergence of block-centered finite differences for elliptic problems, SIAM J. Numer. Anal. 25, pp. 351-375, 1988. 


\begin{tabular}{|c|c|c|}
\hline Decomposition & Clock Time (sec) & $e_{h} * 10^{2}$ \\
\hline $1 \mathrm{x} 1 \mathrm{x} 1$ & 224.13 & 1.13 \\
\hline $2 \times 1 \times 1$ & 112.07 & 1.11 \\
\hline $1 \times 2 \times 1$ & 111.15 & 1.11 \\
\hline $1 \times 1 \times 2$ & 103.58 & 1.11 \\
\hline $2 \times 2 \times 1$ & 55.16 & 1.09 \\
\hline $2 \times 1 \times 2$ & 51.77 & 1.08 \\
\hline $1 \times 2 \times 2$ & 50.61 & 1.08 \\
\hline $2 \times 2 \times 2$ & 24.61 & 1.06 \\
\hline $4 \times 2 \times 2$ & 12.75 & 1.04 \\
\hline $2 \times 4 \times 2$ & 12.15 & 1.05 \\
\hline $2 \times 2 \times 4$ & 11.82 & 1.04 \\
\hline $4 \times 4 \times 2$ & 6.18 & 1.03 \\
\hline $4 \times 2 \times 4$ & 5.95 & 1.02 \\
\hline $2 \times 4 \times 4$ & 5.84 & 1.03 \\
\hline
\end{tabular}

